\title{
Determination of the Fast-Ion Phase-Space Coverage for the FILD Spatial Array of the ASDEX Upgrade Tokamak
}

\author{
J. Ayllon-Guerolaa,b, ${ }^{\star}$, L. Garcia-Baquero ${ }^{b}$, J. Galdon-Quirogac ${ }^{c}, M^{2}$ Garcia- \\ Munoz $^{\mathrm{b}, \mathrm{d}}$, L. Stipani ${ }^{\mathrm{e}}$, J. Gonzalez-Martin ${ }^{\mathrm{a}, \mathrm{b}}$, J. Rivero-Rodriguez ${ }^{\mathrm{a}, \mathrm{b}}$, M. Rodriguez- \\ Ramos $^{b}$, 'L. Sanchis-Sanchez ${ }^{b, d}$, J. Garcia Lopez $^{\mathrm{b}, d}$, A. Herrmann ${ }^{c}$ and the ASDEX \\ Upgrade Team \\ ${ }^{a}$ Department of Mechanical Engineering and Manufacturing, \\ University of Seville, Spain \\ ${ }^{b}$ CNA (U. Sevilla, CSIC, J. de Andalucia), \\ Seville, Spain \\ ${ }^{c}$ Max-Planck-Institut für Plasmaphysik, \\ Garching, Germany \\ ${ }^{d}$ Department of Atomic, Molecular and Nuclear Physics, \\ Faculty of Physics, University of Seville, Spain \\ ${ }^{e}$ Ecole Polytechnique Federale de Lausanne (EPFL), Centre de Recherches en Physique des Plasmas, \\ Association Euratom-Confederation Suisse, Lausanne, Switzerland,
}

E-mail: jayllondus.es

ABSTRACT: The fast-ion phase-space coverage of the ASDEX Upgrade Fast-Ion Loss Detectors (FILD) has been estimated and the results are presented here. To that end, a numerical tool has been developed to determine particle orbits that can be accepted by each detector in the machine, depending on their radial position, without colliding to the first wall. Two different plasma shapes have been analyzed and results show excellent phase-space coverage for mostly all the detectors, especially for those located at the midplane and below it. The methodology developed provides an excellent way to estimate the signal of these detectors depending on the plasma scenario and can be easily extended to other machines. Also, this study can be complemented with thermal analysis to consider the structural integrity of the detectors, allowing determining optimal operation parameters both from the signal and safety standpoints.

KEYWORDS: Fast Ions; Plasma diagnostics; Tokamak; FILD.

\footnotetext{
* Corresponding author.
} 


\section{Contents}

1. Introduction 1

2. The spatial array of FILDs in ASDEX Upgrade 1

3. Phase-space coverage estimation 2

4. Results 4

5. Conclusions and future work 5

\section{Introduction}

In magnetic confinement fusion devices, such as tokamaks and stellarators, proper fast-ion confinement is essential not only because they represent an important energy and momentum source for the plasma [1], but also because their losses, when sufficiently localized, can damage the plasma facing components [2].

Fast-ions losses are extensively studied in many of the major fusion devices by means of fast-ion loss detectors (FILD) [3-7]. This scintillator-based diagnostic allows characterizing the velocity-space of the escaping particles, providing high spatial and temporal resolution [8]. This capability is especially interesting for analyzing the mechanisms responsible for these losses, such as magnetohydrodynamic (MHD) instabilities [9] and externally applied magnetic perturbations $[10,11]$.

The ASDEX Upgrade tokamak (AUG) is equipped with several FILDs, and their combined operation allows obtaining spatially distributed measurements of fast-ion losses. This is of major importance for the 3D analysis of the MHD phenomena related to these losses. The estimation of the fast-ion phase-space covered by the array of FILDs in AUG is the objective of the work presented here.

This paper is structured as follows: in section 2, the spatial array of FILD detectors in AUG is described. Next, in section 3, the simulations performed to characterize the phase-space covered by the FILD array are explained. Finally, in section 4 the results obtained from the simulations are presented and discussed.

\section{The spatial array of FILDs in ASDEX Upgrade}

The AUG Tokamak is equipped with five FILDs located at different toroidal and poloidal positions, as shown in Figure 1. As can be seen in this figure, FILD1 [3] and FILD2 are positioned at the same poloidal position, slightly above the midplane, but at different toroidal angles. FILD4 [12] and FILD5 [13,14], however, are installed below the midplane, FILD5 being very close to the divertor. Finally, FILD3 is located close to the upper divertor. 
During the operation of FILD1, the detector is mounted on the general purpose midplane manipulator of the machine, while the other detectors (FILD2 to FILD5), have their own reciprocating systems. This allows to configure the relative position of each probe with respect to the plasma separatrix. Therefore, the FILDs spatial array can be adapted to a wide variety of plasma shapes, being able to probe a large volume of the escaping fast-ions phase-space.

a)

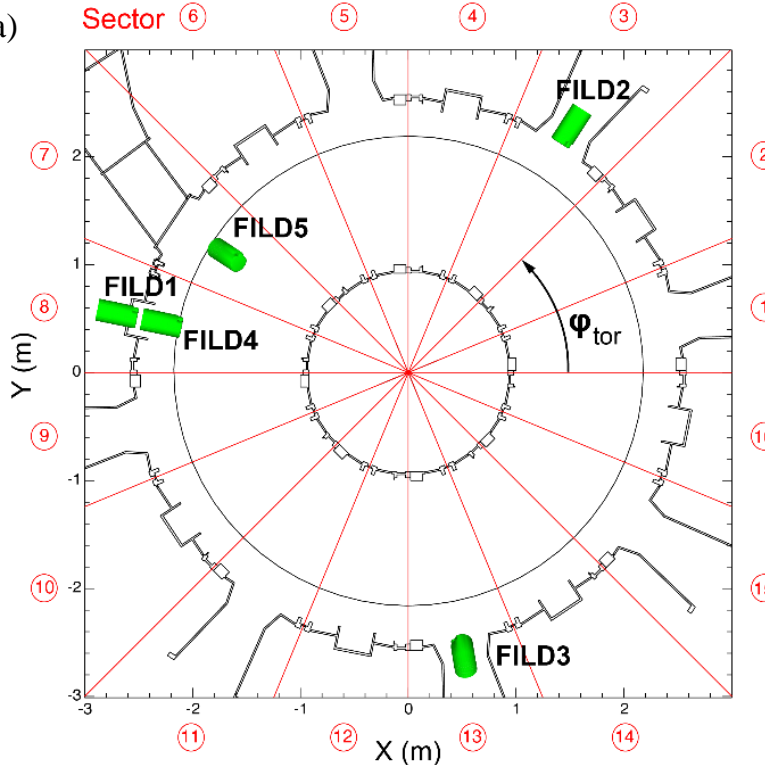

b)

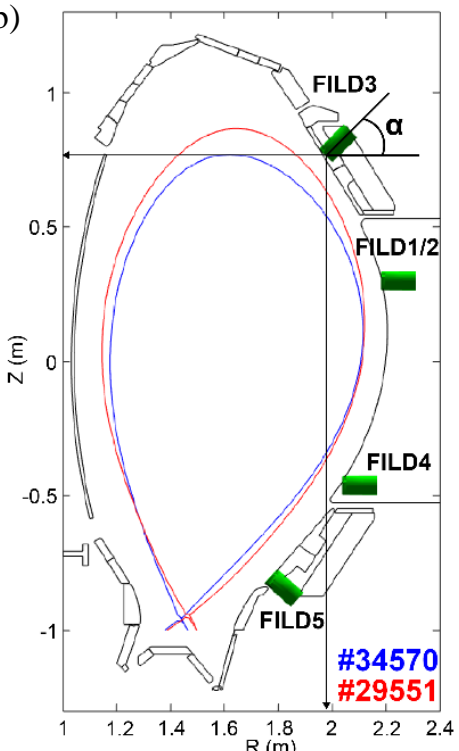

Figure 1. Spatial distribution of FILDs in AUG: a)toroidal view and b)poloidal view. In b), the plasma separatix for two AUG shots, \#34570 (blue) and \#29551 (red), is represented

In Table 1, the main parameters determining the position of each detector in AUG are summarized. The values specified in this table correspond to the positions illustrated in Figure 1 , where the definition of the coordinates $\mathrm{R}, \mathrm{Z}, \varphi_{\text {tor }}$ and $\alpha$ is also provided. The positions shown in Figure 1, where all the probe heads of the detectors are completely hidden behind the plasma limiters, will be considered as the zero-reference for the detector insertion during the simulations described in the next section.

Table 1 - Position parameters of the FILDs array in AUG.

\begin{tabular}{|c|c|c|c|c|c|}
\hline & FILD1 & FILD2 & FILD3 & FILD4 & FILD5 \\
\hline Sector & 8 & 3 & 13 & 8 & 7 \\
\hline $\mathbf{R}(\mathbf{m m})$ & 2180 & 2180 & 1975 & 2035 & 1772 \\
\hline $\mathbf{Z}(\mathbf{m m})$ & 300 & 300 & 765 & -462 & -798 \\
\hline $\boldsymbol{\varphi}_{\text {tor }}(\mathbf{d e g})$ & 169.75 & 57.25 & 282.25 & 169.75 & 147.25 \\
\hline $\boldsymbol{\alpha}(\mathbf{d e g})$ & 0 & 0 & 72 & 0 & -45 \\
\hline
\end{tabular}

\section{Phase-space coverage estimation}

To determine the fast-ion phase-space accessible to a FILD, it is important to consider the working principle of this detector [3,7], which operates close to the plasma separatrix collecting escaping ions. Particles reaching the probe head are collimated and only a fraction of them are allowed to enter through a pinhole and to reach a scintillator plate. Kinetic simulations allow determining the velocity-space of the particles as a function of the position of the strike points. 
The design of the collimator determines the spatial resolution in the scintillator plate hence the velocity-space resolution of FILD, i.e., what particle trajectories will be accepted depending on their pitch angle and energy. Therefore, optimized collimators can be designed according to the characteristics of the target particles, which normally correspond to energies related to plasma heating methods such as neutral beam injectors (NBI) or radiofrequency heating (RF).

Independently of the collimator design, it is useful to determine the potential phase-space that could be covered by the detector, depending on its radial position and the plasma shape. That is, for a given magnetic equilibrium, finding particle trajectories that could eventually reach the collimator pinhole as a function of their energy and pitch angle. This will enhance the operation of FILD, allowing for an optimal positioning of the probe head to provide maximum signal.

To that end, full orbit simulations have been performed to determine, depending on the radial position of the FILDs in the array of AUG, whether the particles can reach the collimator pinhole or, on the contrary, if they are blocked by any of the protruding structures of the first wall. Taking as a reference the position illustrated in Figure 1, each detector has been sequentially inserted approaching the plasma for two different magnetic equilibria, also shown in this figure.

For each detector and insertion, particles are initiated at the centre of the collimator pinhole and tracked back to the plasma for different values of pitch angle and gyroradius. The gyrophase of each trajectory has been adjusted to assure that the particle enters the collimator with the velocity contained in a plane perpendicular to the pinhole. The gyrophase has been considered a fixed parameter during the simulations given that, according to the simulations, its impact on the results is marginal.

Each simulated orbit has been tracked back during one toroidal turn and labelled as "accepted" if no collisions to the wall are identified. Trapped orbits not colliding to the wall have also been considered as accepted. A detailed 2D section of the AUG first wall (shown in Figure 1) has been used to detect blocked orbits. In Figure 2, an accepted trajectory (trapped orbit) is represented for FILD4 and a blocked one for FILD1.
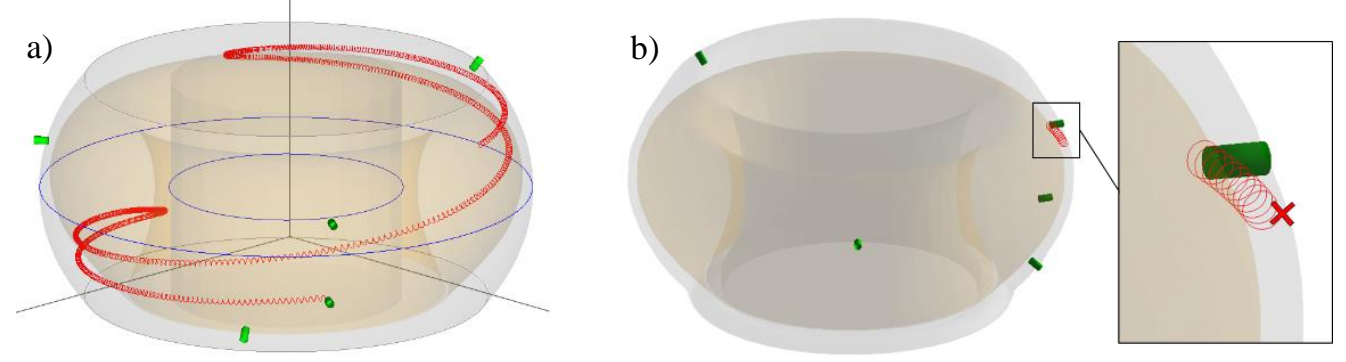

Figure 2. Accepted (a) and blocked (b) orbits for FILD4 and FILD1 respectively.

\section{Results}

The results of the simulations described in the previous section are shown in Figure 3 for two different equilibria, illustrated in Figure 1, corresponding to AUG discharges \#34570 $\left(B_{t}=-\right.$ $\left.2.472 \mathrm{~T}, I_{P}=0.600 \mathrm{MA}, q_{95}=7.023, n_{e}=3.81 \cdot 10^{19} \mathrm{~m}^{-3}\right)$ and $\# 29551\left(B_{t}=-1.813 \mathrm{~T}, I_{P}=\right.$ $0.800 \mathrm{MA}, q_{95}=3.873, n_{e}=6.45 \cdot 10^{19} \mathrm{~m}^{-3}$ ). These contour plots represent, for each detector, the minimum insertion (with respect to the limiter) needed to make the pinhole of the collimator 
accessible to specific orbit gyroradii and pitch angles. The gyroradii associated to the energies of the NBI boxes in AUG are also represented for both equilibria as a reference.

Taking into account the geometry of the probe head, each detector needs to be inserted at least $15 \mathrm{~mm}$ to expose the collimator pinhole (this is the distance between the collimator pinhole and the probe head tip). Therefore, insertions below $15 \mathrm{~mm}$ lead to no phase-space coverage. In general for each detector, and as expected, the phase-space coverage increases as the insertion does, provided that particles are not blocked by the first wall.

As can be observed in Figure 3a) and 3e), FILD1 and FILD2 show very good coverage within the analyzed gyroradius and pitch angle ranges. It is important to clarify here that the phase-space coverage estimated for both detectors is identical given that their poloidal position is coincident and considering the 2D nature of the analysis. The gyroradii associated to the two NBI boxes in AUG are also represented in these figures as a reference.

Figures 3c), 3d), 3g) and 3h) show that excellent phase-space coverage is found for FILD systems below midplane (FILD4 and FILD5) due to favourable ion gradB-drift. For the two equilibria considered in this study, these detectors cover the entire analyzed phase-space ranges with minimal insertion, showing great versatility and, therefore, being able to operate in a wide variety of plasma shapes.

Finally, it is worth mentioning that, due to its position close to the upper divertor and as can be seen in Figures 3b) and 3f), the use of FILD3 is restricted to specifically designed discharges with large plasma elongation and larger insertions are needed to make the fast-ion populations accessible to the detector.

\section{Conclusions and future work}

The potential phase-space of fast-ions covered by the AUG FILDs array has been estimated as a function of the plasma scenario and the radial position of each detector. Full orbit simulations have been performed to detect particle trajectories that could eventually be measured by the FILDs depending on their pitch angle and gyroradius.

Results show that, as expected, reducing the distance from the detectors to the separatrix, increases the phase-space covered by the array. Also, it is shown that the combined operation of the detectors provides a synergetic effect contributing to increase the total phase-space coverage. It is important to clarify here that, during the experiments in AUG, measurements of fast-ion losses have been observed in the FILD detectors with insertions smaller than the minimum insertion provided by the simulations for each detector. The reason for this discrepancy between the simulations and the experimental observations is expected to be associated to the $2 \mathrm{D}$ nature of this analysis (see section 3 ), given that $2 \mathrm{D}$ equilibria and a $2 \mathrm{D}$ vacuum vessel model have been considered. However, the numerical tool developed for this work can be used for qualitatively planning the experiments aiming for an optimal configuration of the array to provide maximum relevant information.

Since this study only considers particles reaching the collimator pinhole, it will be necessary to extend it to include the collimator effect, i.e., tracking the particles up to the scintillator plate. This will provide not only the potential phase-space of the FILDs array but the actual one, according to the specific design of the collimator of each detector. 
a)
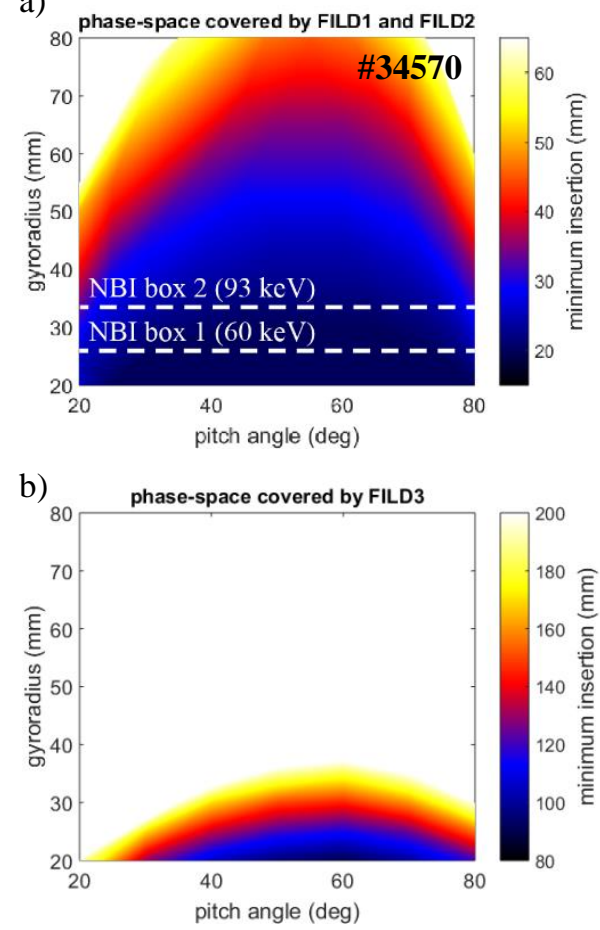

c)

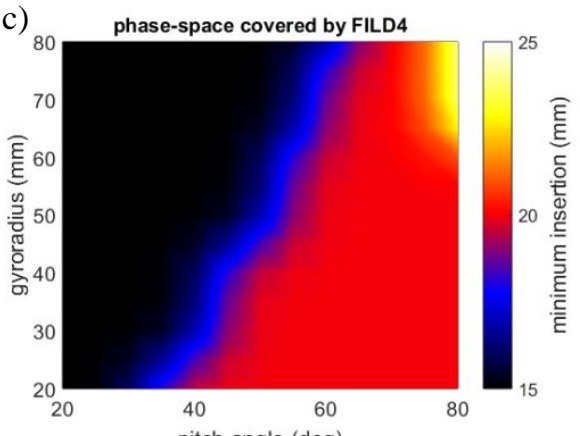

pitch angle (deg)

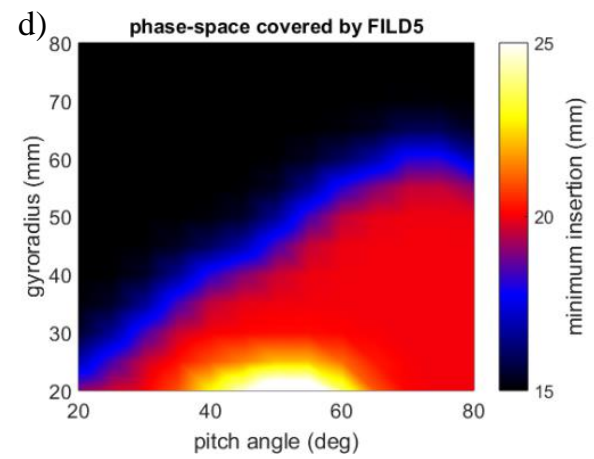

e)
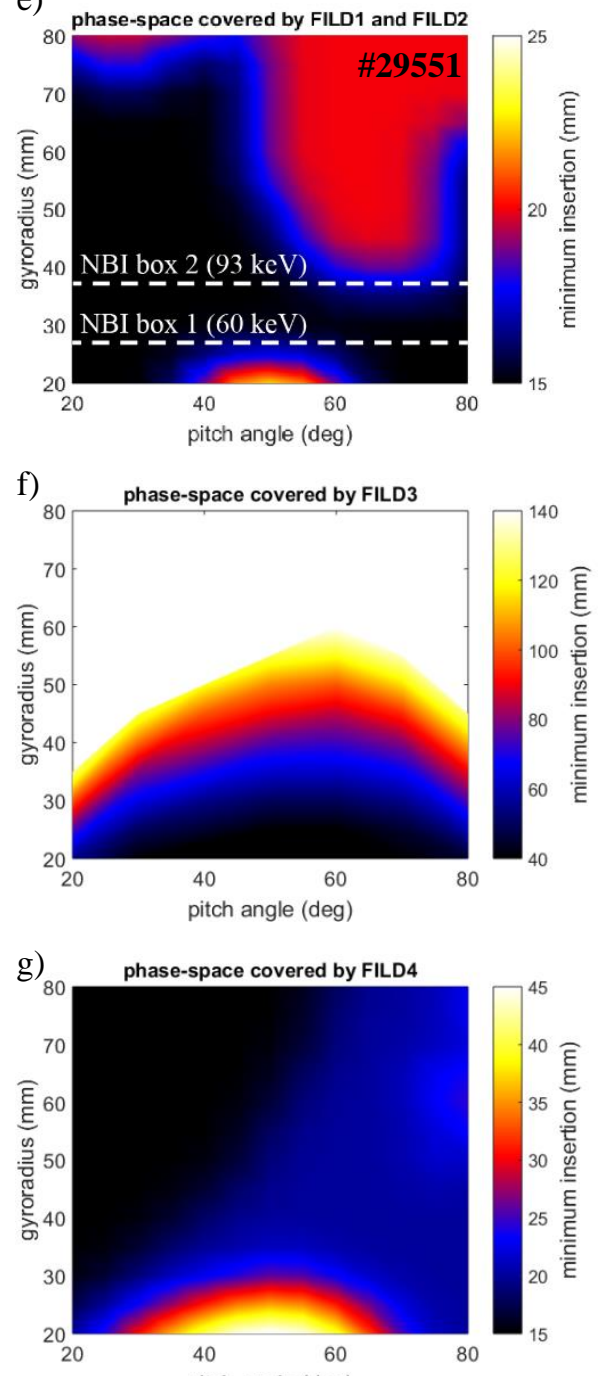

pitch angle (deg)

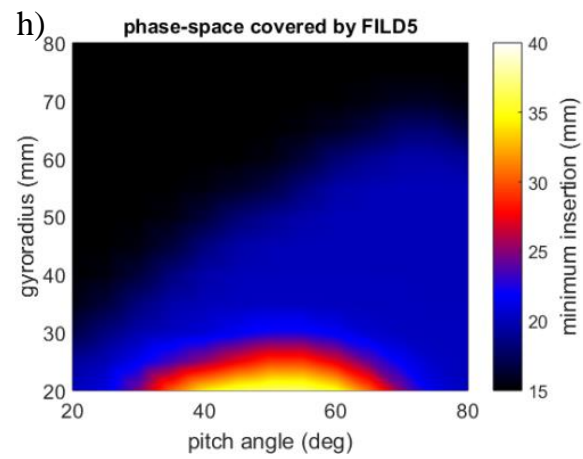

Figure 3. Phase space coverage for the FILDs in the AUG array: For each FILD, the minimum insertion needed to access specific phase-space orbits is represented as a function of the gyroradius and pitch angle of the particle. AUG discharges \#34570 (a-d) and \#29551 (e-h). 
Another important extension of the study presented here is to combine it with an equivalent one in which, depending on the plasma scenario, the thermal load on the probe head is estimated as a function of the radial position of each detector in the array. This will be helpful in obtaining optimal realistic operation parameters considering both the signal expected from the detectors and their structural integrity. This combined study could be easily extended to other machines equipped with FILDs such as K-STAR and DIII-D or machines where FILDs are currently being developed like ITER, JT60SA, MAST-U and TCV.

\section{Acknowledgments}

This research was partly supported by the Spanish Ministry of Economy and Competitiveness (RYC-2011-09152, ENE2012-31087 and FIS2015-69362-P) and a Marie Curie FP7 Integration Grant (PCIG11-GA-2012-321455).

Also, this work has been carried out within the framework of the EUROfusion Consortium and has received funding from the Euratom research and training programme 2014--2018 under grant agreement number 633053 and EUROfusion Engineering Grant ref. EEG-2017/01. The views and opinions expressed herein do not necessarily reflect those of the European Commission.

\section{References}

[1] A. Fasoli et al., Nucl. Fusion 47, S2642 (2007).

[2] J. Galdon-Quiroga et al., Nucl. Fusion 58, 036005 (2018).

[3] M. Garcia-Munoz et al, Rev. Sci. Instrum. 80, 053503 (2009).

[4] S.J. Zweben, Nucl. Fusion 29, 825 (1989).

[5] R. K. Fisher et al., Rev. Sci. Instrum. 81, 10D307 (2010).

[6] J.Y.Kim et al., Rev. Sci. Instrum. 83, 10D305 (2012).

[7] Moseev, D. et al., Rev. Mod. Plasma Phys. 2: 7, (2018).

[8] Galdon-Quiroga et al., Plasma Phys. Control. Fusion 60, 105005 (2018)

[9] M. Garcia-Munoz et al., Phys. Rev. Lett. 104, 185002 (2010).

[10] M. Garcia-Munoz et al., Nucl. Fusion 53, 123008 (2013).

[11] M.A. van Zeeland et al., Plasma Phys. Control. Fusion 56, 015009 (2014).

[12] J. Ayllon-Guerola et al., Rev. Sci. Instrum. 87, $11 \mathrm{E705}$ (2016).

[13] J. Gonzalez-Martin et al., Rev. Sci. Instrum. 89, $10 \mathrm{I106}$ (2018).

[14] J. Gonzalez-Martin et al., submitted to JINST as a proceeding contribution to the $3^{\text {rd }}$ European Conference on Plasma Diagnostics (2019). 